\title{
US Integrated Delivery Networks Perspective on Economic Burden of Patients with Treatment-Resistant Depression: A Retrospective Matched-Cohort Study
}

\author{
Dominic Pilon ${ }^{1} \mathbb{D} \cdot$ Holly Szukis $^{2} \cdot$ Kruti Joshi $^{2} \cdot$ David Singer $^{3} \cdot$ John J. Sheehan ${ }^{2} \cdot$ Jennifer W. Wu $^{1} \cdot$ Patrick Lefebvre $^{1}$. \\ Paul Greenberg ${ }^{4}$
}

Published online: 28 June 2019

(c) The Author(s) 2019

\begin{abstract}
Objective Our objective was to assess healthcare resource utilization (HRU) and costs among patients with major depressive disorder (MDD) with and without treatment-resistant depression (TRD) and those without MDD in US Integrated Delivery Networks (IDNs).

Methods This was a retrospective matched-cohort study. The Optum@ Integrated Claims Electronic Health Record deidentified database was used to identify adult patients with TRD (January 2011-June 2017) across US IDNs. TRD patients were propensity score matched 1:1 with non-TRD MDD and non-MDD patients on demographics. Rates of HRU and costs were compared up to 2 years following the first antidepressant pharmacy claim (or randomly imputed date for non-MDD patients) using negative binomial and ordinary least squares regressions, respectively, with 95\% confidence intervals (CIs) from nonparametric bootstraps (costs only) adjusted for baseline comorbidity index and costs.

Results All 1582 TRD patients were matched to non-TRD MDD and non-MDD patients and evaluated. TRD patients were on average 46 years old, and 67\% were female. Mean duration of observation was 20.1, 19.6, and 17.9 months in the TRD, non-TRD MDD, and non-MDD cohorts, respectively. Patients with TRD had significantly higher rates of HRU than did non-TRD MDD patients (inpatient visits 0.35 vs. 0.16 per patient per year [PPPY]; adjusted incidence rate ratio [IRR] 2.04 [95\% CI 1.74-2.39]) and non-MDD patients (0.35 vs. 0.09 PPPY, adjusted IRR 3.05 [95\% CI 2.54-3.66]). TRD patients incurred significantly higher costs PPPY than did non-TRD MDD patients (\$US25,807 vs. 13,701, adjusted cost difference \$US9479 [95\% CI 7071-11,621]) and non-MDD patients (\$US25,807 vs. 8500, adjusted cost difference \$US11,433 [95\% CI 8668-13,876]).
\end{abstract}

Conclusions HRU and costs associated with TRD are significant in US IDNs.

\section{Introduction}

Major depressive disorder (MDD) is a debilitating psychiatric disorder characterized by a loss of interest in previously enjoyable activities, chronic depression, and suicidal ideations [1]. MDD is one of the most prevalent behavioral health disorders in the United States (US) [2, 3], representing $6.7 \%$ (16.2 million adults) of the overall population [2]. The economic burden associated with MDD was estimated

Electronic supplementary material The online version of this article (https://doi.org/10.1007/s41669-019-0154-z) contains supplementary material, which is available to authorized users.

Dominic Pilon

dominic.pilon@analysisgroup.com

Extended author information available on the last page of the article

\section{Key Points for Decision Makers}

Patients with major depressive disorder (MDD) and treatment-resistant depression (TRD) had higher healthcare resource utilization (HRU) and costs than did patients with MDD who were not resistant to treatment and those without MDD in US Integrated Delivery Networks (IDNs).

Patients with TRD had longer durations of antidepressant therapies and greater variation in antidepressants; effective treatment strategies are urgently needed for this patient population.

The burden of TRD was mainly driven by medicalrelated HRU and costs. 
at \$US210.5 billion in 2010 , with $45-47 \%$ of this burden attributed to direct healthcare costs and $48-50 \%$ due to workplace costs [4].

Antidepressant therapy represents the standard of care for patients with MDD; however, a considerable portion of patients (approximately 30\%) develop treatment-resistant depression (TRD) [5], which is commonly defined as the failure to respond to at least two different antidepressants that are of adequate dose and duration [6, 7]. Although the health and economic burdens associated with MDD are considerable, recent studies have shown that patients with TRD experience an economic burden that is typically twoto-threefold higher than patients who respond to treatment $[8,9]$. A recent study examining direct healthcare costs associated with TRD found that TRD patients had greater healthcare resource utilization (HRU) that resulted in considerably higher direct costs per patient per year (TRD \$US17,261; non-TRD MDD \$US9790; non-MDD \$US4781) [8].

Modification of therapeutic approaches with each failed response requires TRD patients to seek ongoing care from healthcare providers across multiple settings $[6,10,11]$. However, fragmented care resulting from the lack of coordination among different healthcare providers and organizations can interfere with the continuity of measurement-based care required to treat these patients $[12,13]$. To overcome this challenge, collaborative care models such as integrated delivery networks (IDNs) have been proposed [11, 14]. Despite variations in the structure of IDNs, they are broadly defined as healthcare organizations that consist of multiple physicians, hospitals, and other sources of patient care [15-18]. IDNs are increasingly recognized for their capacity to provide quality care across a continuum of settings (e.g., hospitals, health clinics, community health centers) within a single system [11, 19-21].

As organizations dedicated to coordinated patient care, IDNs typically assume financial accountability for patient outcomes, which is often associated with increased efficiency and reduced healthcare costs [11]. Although care in IDNs is expected to decrease costs, conclusive evidence is lacking [15]. IDNs are commonly discussed within the context of improved quality of care [22], yet evidence regarding the costs among MDD and TRD patients treated within US IDNs is sparse. The limited number of reports assessing behavioral health services in collaborative care models $[15,23,24]$ have focused on heterogeneous populations of depressed patients who often have other comorbidities. As a result, these reports may not accurately capture the costs of care among TRD and non-TRD MDD patients. Therefore, this study was conducted to describe the HRU and costs among three cohorts of patients-those with TRD, those with non-TRD MDD, and those with no evidence of MDDwho were treated within IDNs in the US.

\section{Methods}

\subsection{Data Source}

Optum Integrated Claims Electronic Health Records (EHRs) from 1 January 2011 to 30 June 2017 was used for this study. This database included information on $>10$ million individuals insured by UnitedHealth Group with their adjudicated claims linked to Humedica's EHR. Data were de-identified and compliant with the requirements outlined in the Health Insurance Portability and Accountability Act.

\subsection{Study Design}

This retrospective matched-cohort study comprised three mutually exclusive TRD, non-TRD MDD, and non-MDD cohorts. The first antidepressant pharmacy claim (on or after 1 July 2011 for TRD and non-TRD MDD patients, and randomly imputed dates on or after 1 July 2011 for non-MDD patients) was defined as the index date. Patient characteristics were evaluated in the 6 months that preceded the index date (baseline period), and study outcomes (treatment patterns, HRU, and costs) were evaluated from the index date up to 2 years post-index date, the end of continuous enrollment in a health insurance plan, or end of data availability (30 June 2017), whichever occurred first. Since the average time to become TRD was reported to be 1.3 years [6], a 2-year period was used to allow MDD patients the opportunity to be classified as TRD.

Patients with one or more visits to an IDN were considered IDN patients. Once patients were seen in an IDN, they remain flagged as IDN patients even if they later received care in a non-IDN setting. The IDN indicator was not determined at the patient level and was therefore not time or visit specific. Although a consensus definition of an IDN is lacking [15], an IDN was defined as a coordinated hospital inpatient and outpatient system that offers healthcare services in a defined geographic area in the Optum database. Multiple coordinated hospitals or IDNs could be included. IDNs that were included are primarily hospital based in Optum, but they provide more comprehensive services than solely inpatient care. Moreover, Optum defined an IDN as a provider network-level variable; providers self-identified whether they were IDNs and were required to have inpatient data to support their status as IDNs. In this study, $80.1 \%$ of the MDD cohort were associated with an IDN, whereas $75.7 \%$ were associated in the non-MDD cohort (Tables S1-2). 


\subsection{Study Cohorts}

Patients with TRD (TRD cohort) were compared with two control cohorts: patients with MDD who did not have TRD (non-TRD MDD cohort) and patients without MDD (non-MDD cohort). Patients in both control cohorts were propensity score (PS) matched 1:1 with patients in the TRD cohort.

To be included in both the TRD and the non-TRD MDD cohorts, patients were required to meet the following criteria: (1) one or more diagnoses for MDD (International Classification of Diseases, Ninth Edition, Clinical Modification [ICD-9-CM]: 296.2x [MDD - single episode], 296.3x [MDD - recurrent episode]; ICD, Tenth Edition, CM [ICD10-CM]: F32.x [excluding F32.8], F33.x [excluding F33.8]); (2) one or more claims for an antidepressant between 1 July 2011 and 30 June 2017 (MDD diagnosis could occur before or after the first antidepressant pharmacy claim); (3) one or more diagnoses of depression (ICD-9-CM: 296.2x, 296.3x, 300.4x, 311.x, 309.0x, or 309.1x; ICD-10-CM: F32.x, F33.X, F34.1, or F43.21) during the baseline or observation period. Both the baseline and follow-up periods were used to identify patients with depression due to the episodic nature of the disease [25].

\subsubsection{Treatment-Resistant Depression (TRD) Cohort}

In the TRD cohort, a pharmacy claims-based algorithm was used to identify MDD patients who were likely to have TRD within 2 years after the index date (i.e., follow-up). MDD patients were considered likely to have TRD if their MDD did not respond to two antidepressant treatment regimens, including augmentation therapy with an anticonvulsant, anxiolytic, antipsychotic, lithium, psychostimulant, or thyroid hormone medication, with adequate dose and duration (>6 weeks) as per the American Psychiatric Association guidelines [7]. Failure of a treatment regimen was defined as a switch of antidepressant (i.e., $<180$ days after the end of the previous treatment), the addition of an antidepressant, or the initiation of an augmentation therapy. The initiation of the third treatment regimen had to occur $>6$ weeks after the start of the first antidepressant treatment.

\subsubsection{Non-TRD Major Depressive Disorder (MDD) Cohort}

Patients in the non-TRD MDD cohort included MDD patients who did not meet the criteria for TRD, as previously defined, within 2 years of the index date.

\subsubsection{Non-MDD Cohort}

Patients in the non-MDD cohort consisted of a randomly selected sample of patients without MDD (ICD-9-CM: 296.2x, 296.3x; ICD-10-CM: F32.x, F33.x [excluding F33.8]) from 1 January 2011 to 30 June 2017. Since a retrospective matched-cohort study design was used, the random selection of 500,000 non-MDD patients was considered sufficient to provide a pool of controls eligible for matching.

\subsubsection{All Cohorts}

All patients included in this study were required to meet the following conditions: (1) no diagnosis for specific psychiatric comorbidities (i.e., psychosis [ICD-9-CM: 298.xx; ICD-10-CM: F23.x, F25.x, F44.89], schizophrenia [ICD9-CM: 295.xx; ICD-10-CM: F20.x, F25.x], bipolar disorder/manic depression [ICD-9-CM: 296.0x, 296.1x, 296.4x, 296.5x, 296.6x, 296.7x, 296.8x; ICD-10-CM: F30.x, F31.x], dementia [ICD-9-CM: 290.xx, 294.1x; ICD-10-CM: F01.x, F02.x, F03.x]) between 1 January 2011 and 30 June 2017; (2) $\geq 18$ years at the index date; (3) $\geq 6$ months of continuous enrollment in a health insurance plan before and after the index date; (4) no antidepressant claims during the baseline period.

\subsection{Study Measures}

Baseline characteristics included demographics, physical and mental comorbidities, medication use, HRU, and costs were used to assess the distribution of the TRD, non-TRD, and non-MDD cohorts.

For each cohort, the proportion of patients receiving antidepressant medications during the observation period was reported. Duration of antidepressant therapy was also presented and defined as the number of days with medication available between the first antidepressant claim (i.e., index date) and the last day of supply of antidepressant with no gaps $>14$ days (gaps were excluded in duration of therapy).

HRU and costs were categorized as all-cause, behavioral health-related, depression-related, and suicide-related. For each category, HRU and costs were divided into inpatient visits, inpatient days, emergency department (ED) visits, outpatient visits, and other visits. Behavioral health-related medical HRU and costs (including depression-related but not suicide-related costs) were identified using primary or secondary ICD-9-CM diagnostic codes 290.xx-319. $\mathrm{xx}$ and ICD-10-CM diagnostic codes F01.xxx-F99.xxx. Psychiatric pharmacy costs included the following classes of agents: anxiolytics, antidepressants, anticonvulsants/ mood stabilizers, antipsychotics, and other mood stabilizers (e.g., lithium). Depression-related HRU and costs were 
identified using primary or secondary ICD-9-CM diagnostic codes 296.2x, 296.3x, 300.4x, 309.0x, 309.1x, 311.xx and ICD-10-CM diagnostic codes F32.x, F33.x, F34.1, or F43.21. Antidepressant pharmacy costs included the following classes of agents, selective serotonin reuptake inhibitors (SSRIs), norepinephrine-dopamine reuptake inhibitors (NDRIs), serotonin-norepinephrine reuptake inhibitors (SNRIs), serotonin modulators (i.e., nefazodone, trazodone, vilazodone, venlafaxine), tricyclics and tetracyclics, norepinephrine-serotonin modulators, monoamine oxidase inhibitors (MAOIs), and other (i.e., olanzapine-fluoxetine). Suicide-related HRU and costs were defined using primary or secondary ICD-9-CM diagnostic codes E95x or V62.84 and ICD-10-CM diagnostic codes T14.91x, X71.x-X83.x, T36. $x$ - T65. $x$ with a suffix of ' 2 ' indicating 'intentional selfharm', T71. $x$ with a suffix of ' 2 ' indicating 'intentional selfharm' or R45.851.

\subsection{Statistical Analysis}

Patients with TRD were matched 1:1 to non-TRD MDD and non-MDD patients using PS defined as the conditional probability of having TRD based on observable characteristics [26, 27]. The PS across patients were classified into strata, each containing an equal proportion of patients (5\%). One TRD patient was matched to one non-TRD MDD and one non-MDD patient within the same stratum. The logistic regression model was used to estimate the PS, where having TRD (yes/no) was the binary dependent variable and patient characteristics were the predictors and included age, sex, race, year of the index date, geographical region, and type of healthcare plan. Baseline patient characteristics were compared using standardized differences; a covariate with a standardized difference $<10 \%$ was considered well-balanced [26]. Rates of HRU were compared between matched cohorts using multivariable negative binomial regression (i.e., incidence rate ratios [IRRs]). Costs were expressed as per patient per year (PPPY) in \$US, year 2017 values, using the Consumer Price Index for Medical care using means, standard deviations (SDs), and medians, and adjusted cost differences were calculated using multivariable ordinary least squares regression with 95\% confidence intervals (CIs) and p-values obtained from nonparametric bootstraps with 499 replications. To account for censoring in the cost data, a phase-based approach has been recommended in studying cumulative costs [28]. Specifically, mean costs per patient per month (PPPM) in the 6 months before and up to 2 years after the index date and stratified by 1-month periods were calculated as a sensitivity analysis. Since the matching was limited to demographic variables, baseline Quan-Charlson comorbidity index (Quan-CCI) [29] and all-cause healthcare costs were adjusted for in the multivariable models due to the remaining imbalances observed after matching.
Given that TRD is complex, with multifactorial causes and effects with respect to other comorbidities, minimally matching on and controlling for potential confounders to avoid conditioning on mediators was deemed appropriate in this context. For the parameter estimates, standard errors, and Akaike information criterion from the regression models, see Tables S3-7.

\section{Results}

\subsection{Baseline Demographic and Clinical Characteristics}

Among 12,730 treated patients with MDD, 1582 (12\%) met the criteria for TRD (Table S1). A total of 63,828 patients without MDD who met the inclusion criteria were identified (Table S2). Before matching, TRD patients were younger than non-TRD MDD and non-MDD patients (Table S8). After matching, cohorts were well-balanced (standardized difference $<10 \%$ ) on demographic factors (Table 1). TRD patients were more likely to have other behavioral healthrelated medication use during the baseline period, even after matching. See Figs. S1-S2 for PS distributions. The mean duration of the observation period was 20.1, 19.6, and 17.9 months in the TRD, non-TRD MDD, and non-MDD cohorts, respectively.

\subsection{Treatment Patterns}

Patients with TRD used a greater variety of antidepressants than did patients in the other cohorts. In the TRD and non-TRD MDD cohorts, the three most frequently used antidepressants included SSRIs (89 vs. $81 \%$ ), NDRIs (47 vs. $22 \%$ ), and SNRIs (45 vs. $16 \%$; Fig. 1). The use of antidepressants was low in the non-MDD cohort (SSRIs 8\%, NDRIs 2\%, and SNRIs 2\%). The duration of antidepressant therapy was longer among TRD patients than among nonTRD MDD and non-MDD patients (Table S9).

\subsection{Healthcare Resource Utilization (HRU) and Costs}

Patients with TRD had higher rates of HRU during the observation period than did the non-TRD MDD and nonMDD patients (Fig. 2). TRD patients had 2.04 (95\% CI 1.74-2.39) and 3.05 (95\% CI 2.54-3.66) times the rate of inpatient visits for non-TRD MDD and non-MDD patients, respectively. All-cause inpatient length of stay was higher for TRD patients (3.19 days) than for non-TRD (1.04 days) and non-MDD (0.78 days) patients (adjusted IRR 2.98 [95\% CI 2.34-3.81] and 2.43 [95\% CI 1.85-3.20], respectively). Similarly, TRD patients had higher rates of behavioral health-related HRU (e.g., 2.61 [95\% CI 2.23-3.06] 
Table 1 Baseline characteristics, healthcare resource utilization, and all-cause healthcare costs evaluated 6 months prior to the index date

\begin{tabular}{|c|c|c|c|c|c|}
\hline \multirow[t]{3}{*}{ Variables } & \multicolumn{5}{|c|}{ Matched population $^{\mathrm{a}}$} \\
\hline & \multirow{2}{*}{$\begin{array}{l}\text { TRD cohort } \\
N=1582\end{array}$} & \multirow{2}{*}{$\begin{array}{l}\text { Non-TRD MDD cohort } \\
N=1582\end{array}$} & \multirow[t]{2}{*}{ Std. diff. ${ }^{\mathrm{b}}(\%)$} & \multirow{2}{*}{$\begin{array}{l}\text { Non-MDD control cohort } \\
N=1582\end{array}$} & \multirow[t]{2}{*}{ Std. diff. ${ }^{\mathrm{b}}(\%)$} \\
\hline & & & & & \\
\hline Age at index date, years & $45.6 \pm 16.6[45]$ & $45.4 \pm 16.7[44]$ & 0.7 & $44.9 \pm 16.8[44]$ & 4.1 \\
\hline Female & $1052(66.5)$ & $1067(67.4)$ & 2.0 & $1088(68.8)$ & 5.0 \\
\hline \multicolumn{6}{|l|}{ Race } \\
\hline White & $1345(85.0)$ & 1339 (84.6) & 1.1 & $1313(83.0)$ & 5.4 \\
\hline Black & $68(4.3)$ & $69(4.4)$ & 0.3 & $58(3.7)$ & 3.2 \\
\hline Asian & $16(1.0)$ & $15(0.9)$ & 0.6 & $15(0.9)$ & 0.6 \\
\hline Hispanic & $51(3.2)$ & $68(4.3)$ & 5.7 & $76(4.8)$ & 8.1 \\
\hline Other/unknown & $102(6.4)$ & $91(5.8)$ & 2.9 & $120(7.6)$ & 4.5 \\
\hline \multicolumn{6}{|l|}{ Year of index date ${ }^{c}$} \\
\hline 2011 & 215 (13.6) & $203(12.8)$ & 2.2 & $224(14.2)$ & 1.6 \\
\hline 2012 & $266(16.8)$ & $256(16.2)$ & 1.7 & $273(17.3)$ & 1.2 \\
\hline 2013 & $260(16.4)$ & $262(16.6)$ & 0.3 & $286(18.1)$ & 4.4 \\
\hline 2014 & $250(15.8)$ & $243(15.4)$ & 1.2 & $259(16.4)$ & 1.6 \\
\hline 2015 & $292(18.5)$ & 294 (18.6) & 0.3 & $291(18.4)$ & 0.2 \\
\hline 2016 & $246(15.5)$ & $274(17.3)$ & 4.8 & $249(15.7)$ & 0.5 \\
\hline 2017 & $53(3.4)$ & $50(3.2)$ & 1.1 & $0(0.0)$ & 25.9 \\
\hline \multicolumn{6}{|l|}{ Geographical region $^{\mathrm{d}}$} \\
\hline Northeast & $160(10.1)$ & $163(10.3)$ & 0.6 & $163(10.3)$ & 0.6 \\
\hline Midwest & $792(50.1)$ & $768(48.5)$ & 3.0 & 807 (51.0) & 1.9 \\
\hline South & $396(25.0)$ & $419(26.5)$ & 3.3 & 378 (23.9) & 2.6 \\
\hline West & $191(12.1)$ & $178(11.3)$ & 2.6 & 185 (11.7) & 1.2 \\
\hline Unknown & $43(2.7)$ & $54(3.4)$ & 4.0 & $49(3.1)$ & 2.3 \\
\hline \multicolumn{6}{|l|}{ Type of healthcare plan } \\
\hline Medicare & $283(17.9)$ & $259(16.4)$ & 4.0 & $254(16.1)$ & 4.9 \\
\hline Commercial & $1299(82.1)$ & $1323(83.6)$ & 4.1 & $1328(83.9)$ & 5.0 \\
\hline Preferred provider organization & $78(4.9)$ & $85(5.4)$ & 2.0 & $84(5.3)$ & 1.7 \\
\hline Point of service plan & 907 (57.3) & 908 (57.4) & 0.1 & $955(60.4)$ & 6.3 \\
\hline Health management organization & $189(11.9)$ & $187(11.8)$ & 0.4 & $176(11.1)$ & 2.6 \\
\hline Exclusive provider organization & $84(5.3)$ & $93(5.9)$ & 2.5 & $71(4.5)$ & 3.8 \\
\hline Other healthcare plan & $41(2.6)$ & $50(3.2)$ & 3.4 & $42(2.7)$ & 0.4 \\
\hline Quan-CCI [29] & $0.6 \pm 1.3[0]$ & $0.5 \pm 1.1[0]$ & 12.1 & $0.3 \pm 0.9[0]$ & 29.0 \\
\hline $\begin{array}{l}\text { Number of unique mental health } \\
\text { diagnoses }\end{array}$ & $1.2 \pm 1.6[1]$ & $0.9 \pm 1.3[0]$ & 20.8 & $0.2 \pm 0.5[1]$ & 84.3 \\
\hline $\begin{array}{l}\text { Other mental health-related medi- } \\
\text { cation use }[42,43]^{\mathrm{e}}\end{array}$ & $614(38.8)$ & $343(21.7)$ & 35.2 & $152(9.6)$ & 62.4 \\
\hline \multicolumn{6}{|c|}{ Top five most frequent physical comorbidities $[44]^{\mathrm{f}}$} \\
\hline Hypertension & $405(25.6)$ & $338(21.4)$ & 9.9 & $243(15.4)$ & 24.6 \\
\hline Diabetes & $196(12.4)$ & $147(9.3)$ & 9.9 & $92(5.8)$ & 22.5 \\
\hline Chronic pulmonary disease & $167(10.6)$ & $157(9.9)$ & 2.1 & $94(5.9)$ & 16.6 \\
\hline Obesity & $127(8.0)$ & $128(8.1)$ & 0.2 & $78(4.9)$ & 12.5 \\
\hline Hypothyroidism & $140(8.8)$ & $121(7.6)$ & 4.4 & $100(6.3)$ & 9.5 \\
\hline \multicolumn{6}{|c|}{ Top five most frequent mental comorbidities ${ }^{\mathrm{g}}$} \\
\hline Depression $^{\mathrm{h}}$ & $556(35.1)$ & $478(30.2)$ & 10.3 & $37(2.3)$ & 76.7 \\
\hline Anxiety disorders & $345(21.8)$ & $245(15.5)$ & 15.9 & $70(4.4)$ & 49.1 \\
\hline Sleep-wake disorders & $220(13.9)$ & $154(9.7)$ & 12.8 & $65(4.1)$ & 33.3 \\
\hline $\begin{array}{l}\text { Substance-related and addictive } \\
\text { disorders }\end{array}$ & 188 (11.9) & $116(7.3)$ & 15.3 & $55(3.5)$ & 30.9 \\
\hline
\end{tabular}


Table 1 (continued)

\begin{tabular}{|c|c|c|c|c|c|}
\hline \multirow[t]{3}{*}{ Variables } & \multicolumn{5}{|l|}{ Matched population $^{\mathrm{a}}$} \\
\hline & \multirow{2}{*}{$\begin{array}{l}\text { TRD cohort } \\
N=1582\end{array}$} & \multirow{2}{*}{$\begin{array}{l}\text { Non-TRD MDD cohort } \\
N=1582\end{array}$} & \multirow[t]{2}{*}{ Std. diff. ${ }^{\mathrm{b}}(\%)$} & \multirow{2}{*}{$\begin{array}{l}\text { Non-MDD control cohort } \\
N=1582\end{array}$} & \multirow[t]{2}{*}{ Std. diff. ${ }^{\text {b }}(\%)$} \\
\hline & & & & & \\
\hline $\begin{array}{l}\text { Other conditions that may be a } \\
\text { focus of clinical attention }\end{array}$ & $121(7.6)$ & $110(7.0)$ & 2.7 & $65(4.1)$ & 14.9 \\
\hline \multicolumn{6}{|l|}{$\mathrm{Had} \geq 1$ healthcare visit/service } \\
\hline Inpatient & $217(13.7)$ & $144(9.1)$ & 14.3 & $57(3.6)$ & 35.0 \\
\hline $\mathrm{ED}$ & $203(12.8)$ & $178(11.3)$ & 4.8 & $112(7.1)$ & 18.9 \\
\hline Outpatient & $1398(88.4)$ & $1342(84.8)$ & 9.8 & $1180(74.6)$ & 30.3 \\
\hline Other & $366(23.1)$ & $306(19.3)$ & 9.2 & $193(12.2)$ & 27.8 \\
\hline $\begin{array}{l}\text { All-cause healthcare costs (\$US, } \\
\text { year } 2017 \text { values) }\end{array}$ & $21,872 \pm 61,869[4937]$ & $13,696 \pm 44,271[2643]$ & 15.2 & $7160 \pm 24,755[1350]$ & 31.2 \\
\hline Medical costs & $19,802 \pm 60,601[3363]$ & $12,357 \pm 43,421[1740]$ & 14.1 & $5918 \pm 23,754[868]$ & 30.2 \\
\hline Pharmacy costs & $2070 \pm 6719[420]$ & $1339 \pm 4201[230]$ & 13.0 & $1242 \pm 5943[105]$ & 13.1 \\
\hline
\end{tabular}

Data are presented as $n(\%)$ or mean \pm standard deviation [median] unless otherwise indicated

$E D$ emergency department, $I C D-x-C M$ International Classification of Diseases, $x$ th edition, Clinical Modification, $M D D$ major depressive disorder, Quan-CCI Quan-Charlson comorbidity index, SD standard deviation, Std. diff. standardized difference, TRD treatment-resistant depression

${ }^{\text {a }}$ Patients were matched on propensity score (the probability of being in the TRD cohort vs. the non-TRD MDD or non-MDD cohort), modelled using a logistic regression model adjusted for categorical age, sex, race, year of the index date, geographical region, and type of healthcare plan

${ }^{b}$ For continuous variables, the std. diff. was calculated by dividing the absolute difference in means of the control and the TRD cohorts by the pooled SD of both groups. The pooled SD is the square root of the average of the squared SDs. For dichotomous variables, the std. diff. was calculated using the following equation, where $\mathrm{P}$ is the respective proportion of participants in each group: (PTRD $-\mathrm{Pcontrol}) / \sqrt{ }[(\mathrm{PTRD}(1-\mathrm{PTR}$ D) + Pcontrol $(1-$ Pcontrol $)) / 2]$

${ }^{c}$ For TRD and non-TRD MDD patients, the index date was defined as the date of the first prescription fill for an antidepressant. For non-MDD patients, the index date was randomly generated

${ }^{\mathrm{d}}$ Based on US census regions (http://www2.census.gov/geo/pdfs/maps-data/maps/reference/us_regdiv.pdf)

${ }^{\mathrm{e}}$ Includes anxiolytics, anticonvulsants/mood stabilizers, antipsychotics, psychostimulants, thyroid hormone (T3), and lithium. Agents were grouped according to their generic name

${ }^{\mathrm{f}}$ The top five most frequent Elixhauser comorbidities identified in the TRD cohort were reported

${ }^{\mathrm{g}}$ The top five most frequent mental disorders identified in the TRD cohort were reported

${ }^{\text {h}}$ Depression diagnoses included the following diagnoses ICD-9-CM: 296.2x (MDD—single episode), 296.3x (MDD—recurrent episode), 300.4x (dysthymic disorder), 309.0x (adjustment disorder with depressed mood), 309.1x (prolonged depressive reaction), and 311.x (depressive disorder, not elsewhere classified) or ICD-10-CM: F32x (MDD—single episode), F33x (MDD—recurrent episode), F341 (dysthymic disorder) and F4321 (adjustment disorder with depressed mood). All patients had to have a diagnosis of MDD during the study period, but only a portion had an MDD diagnosis during the baseline period

and 17.44 [95\% CI 11.62-26.16] times the rate of inpatient visits for TRD patients vs. non-TRD MDD and non-MDD patients, respectively); the rate of depression- and suiciderelated inpatient visits was 2.48 (95\% CI 1.98-3.11) and 3.26 (95\% CI 2.28-4.68), respectively, times higher for the TRD than for the non-TRD MDD patients (Table S10).

During the baseline period, the mean all-cause healthcare cost PPPY was \$US21,872, \$US13,696, and \$US7160 for TRD, non-TRD MDD, and non-MDD patients, respectively (Table 1). For the TRD and non-TRD MDD cohorts, allcause medical costs were \$US19,802 and 12,357, respectively, which accounted for $>90 \%$ of all-cause healthcare costs. During the follow-up period, the unadjusted cost differences PPPY illustrated that all-cause healthcare costs for the TRD cohort were $88 \%$ and $304 \%$ higher than for the non-TRD MDD and non-MDD cohorts (\$US25,807 [TRD] vs. 13,701 [non-TRD MDD] or 8500 [non-MDD]; Table 2). After adjusting for baseline Quan-CCI and allcause healthcare costs, TRD patients had significantly higher PPPY all-cause healthcare costs than did non-TRD MDD and non-MDD patients (\$US9479 [95\% CI 7071-11,621] more than the non-TRD MDD cohort and \$US11,433 [95\% CI 8668-13,876] more than the non-MDD cohort; Fig. 3). Similarly, TRD patients had the highest all-cause inpatient costs (\$US3805 [95\% CI 2289-5268] more than the nonTRD MDD cohort and \$US4199 [95\% CI 2566-5484] more than the non-MDD cohort), which were the main drivers of the adjusted cost difference (Table 2). Additionally, mean 


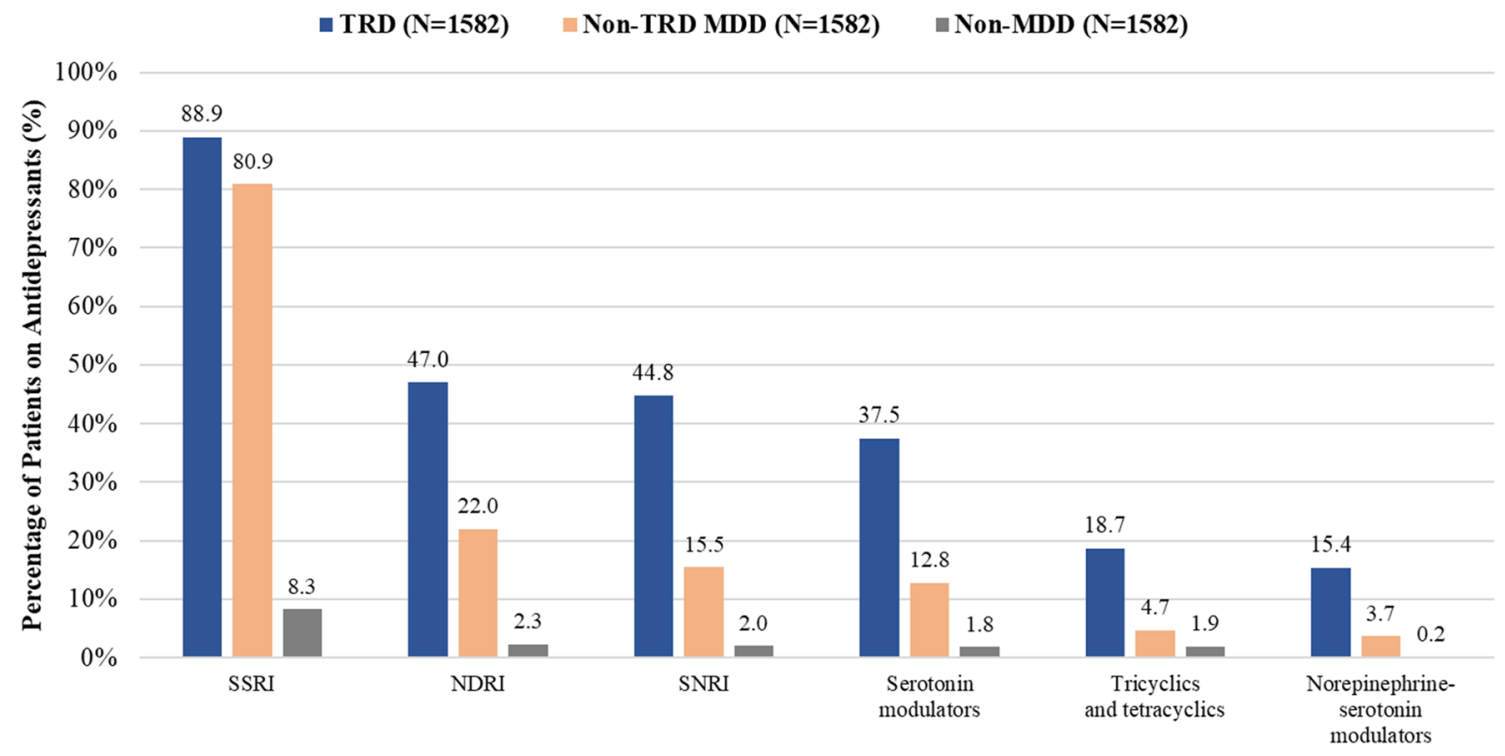

Fig. 1 Medication treatment patterns during the observation period among TRD, non-TRD MDD, and non-MDD cohorts. $M D D$ major depressive disorder, NDRIs norepinephrine-dopamine reuptake inhibitors, SNRIs serotonin-norepinephrine reuptake inhibitors, SSRIs selective serotonin reuptake inhibitors, TRD treatment-resistant depression

\section{(a) TRD cohort versus nonTRD MDD cohort}

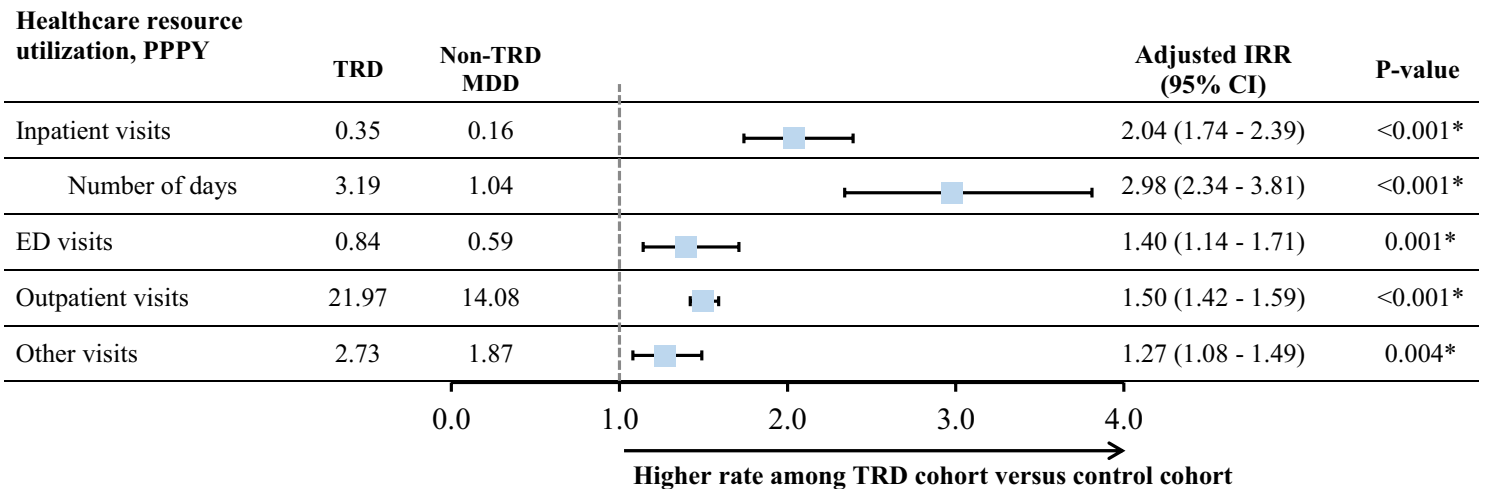

(b) TRD cohort versus non-MDD cohort

\begin{tabular}{|c|c|c|c|c|c|c|}
\hline $\begin{array}{l}\text { Healthcare resource } \\
\text { utilization, PPPY }\end{array}$ & TRD & Non-MDD & & & $\begin{array}{c}\text { Adjusted IRR } \\
(95 \% \text { CI) }\end{array}$ & P-value \\
\hline Inpatient visits & 0.35 & 0.09 & & $\longmapsto$ & $3.05(2.54-3.66)$ & $<0.001^{*}$ \\
\hline Number of days & 3.19 & 0.78 & & $\longmapsto$ & $2.43(1.85-3.20)$ & $<0.001^{*}$ \\
\hline ED visits & 0.84 & 0.42 & & $\longmapsto$ & $1.86(1.50-2.31)$ & $<0.001 *$ \\
\hline Outpatient visits & 21.97 & 8.16 & & $r-1$ & $2.66(2.49-2.84)$ & $<0.001^{*}$ \\
\hline \multirow[t]{2}{*}{ Other visits } & 2.73 & 0.91 & & $\longmapsto$ & $2.29(1.93-2.73)$ & $<0.001^{*}$ \\
\hline & & 0.0 & 1.0 & 3.0 & 4.0 & 30 \\
\hline
\end{tabular}

Higher rate among TRD cohort versus control cohort

Fig. 2 Healthcare resource utilization measured from the index date up to 2 years post-index date ${ }^{\mathrm{a}}$. ${ }^{\mathrm{a}}$ Multivariable negative binomial regression included baseline all-cause healthcare costs and QuanCCI. *Significant at the $5 \%$ level. $C I$ confidence interval, ED emer- gency department, $H R U$ healthcare resource utilization, IRR incidence rate ratio, $M D D$ major depressive disorder, $P P P Y$ per patient per year, Quan-CCI Quan-Charlson comorbidity index, TRD treatment-resistant depression 


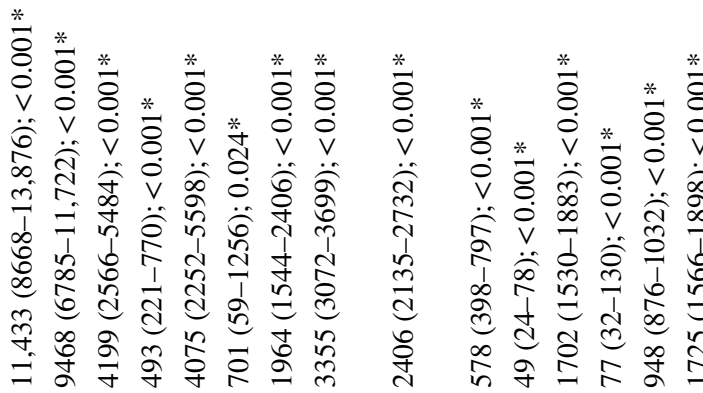

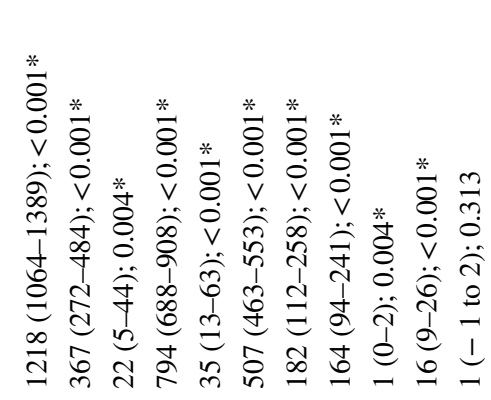

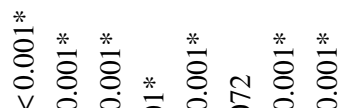

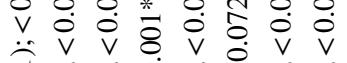

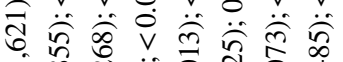

च

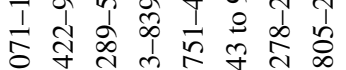

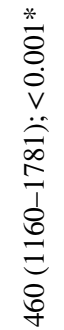

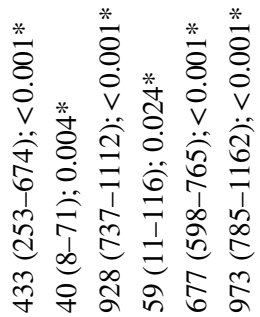

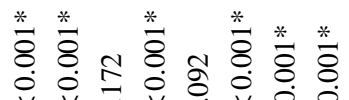

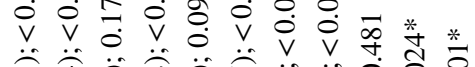

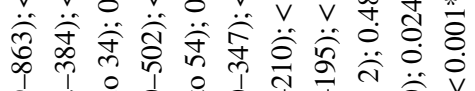

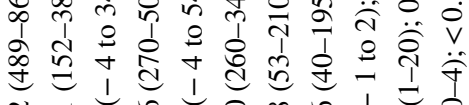

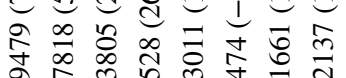

水

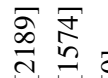

듐

光

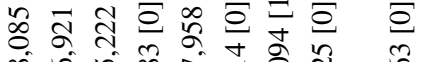

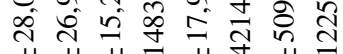

훙ㅎㅇㅎㅇㅎㅇ $\bar{c}$

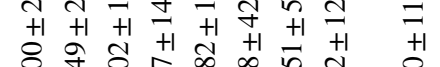

ले

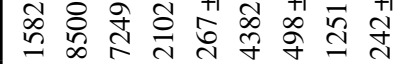

in $\underset{+1}{+1} \stackrel{+1}{=} \stackrel{+1}{+1} \stackrel{+}{a}$

훙ㅎㅎㅎㅇㅎㅇ

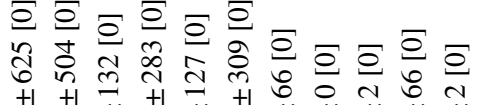

ते

ॠ㤀

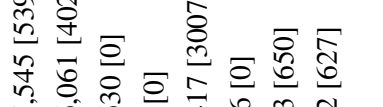

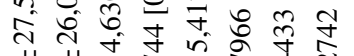

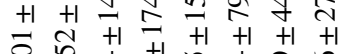

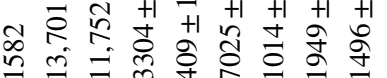

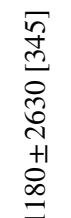

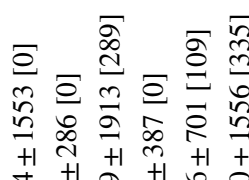

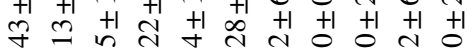

$\bar{\Xi}$

$+1+\lambda$ in +1 व $\infty$ ह

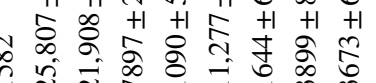

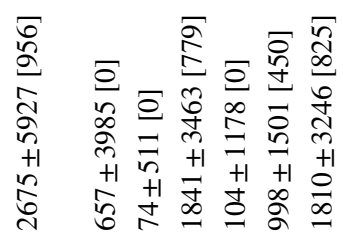

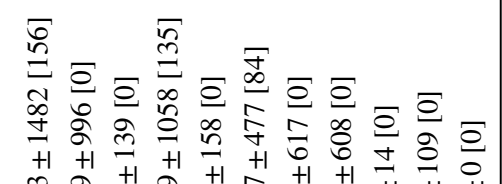

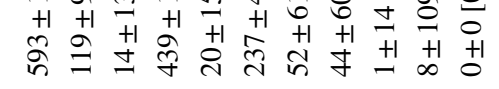

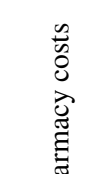<smiles>C1CCC2(CC1)CCCC2</smiles>

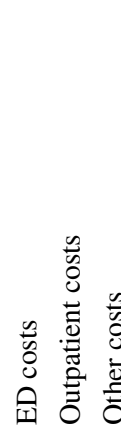

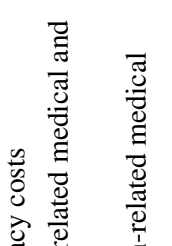

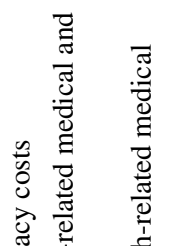

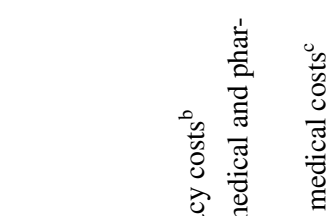

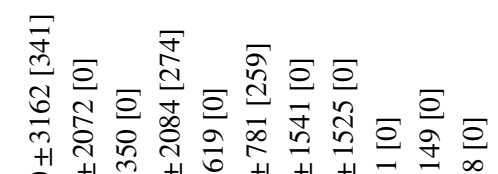

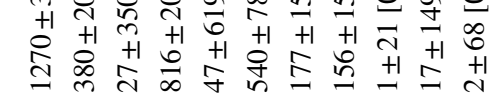

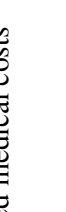


costs PPPM in the 6 months before and up to 2 years after the index date and stratified by a 1-month period are presented in Fig. S3. Although there was some variability, mean costs PPPM were on average higher in TRD than non-TRD MDD cohorts (\$US1762-2863 vs. 839-1757) each month.

\section{Discussion}

The results of this retrospective matched-cohort analysis demonstrated that patients with TRD in IDN settings across the US had a longer duration of antidepressant therapy and a greater number of unique antidepressant medications than patients with MDD that were not resistant to treatment and than patients without MDD. From baseline to follow-up, TRD patients had higher costs and rates of HRU than did non-TRD MDD and non-MDD patients. All-cause medical and pharmacy costs were also higher, on average, among TRD patients. During the baseline period, TRD patients had higher rates of HRU, particularly behavioral health-related HRU than did non-TRD MDD patients and non-MDD patients. TRD patients also had higher healthcare costs, with inpatient costs serving as the main driver of this increased adjusted cost difference.

In this study, healthcare costs were considerably high and contrary to expectations that IDNs are associated with lower costs [11, 15, 30]. Instead, the higher HRU and costs among TRD patients relative to non-TRD MDD or nonMDD patients in this study aligned with previous commercial claims-based studies of patients treated in non-IDN settings $[6,8,9,31,32]$. A recent study, by Amos et al. [8], evaluated HRU and costs among commercially insured TRD patients using a similar study design as the present study, which allows for indirect comparisons. Results of that analysis demonstrated that the rates of HRU for TRD patients were higher than rates for patients in the non-TRD MDD and non-MDD cohorts (2.0 and 4.7 times the rate of inpatient visits versus the non-TRD MDD and nonMDD cohorts, respectively; all $p<0.001$ ). As in our study, TRD patients also incurred significantly higher healthcare costs PPPY than non-TRD MDD patients and non-MDD patients (\$US6709 more than the non-TRD MDD cohort and \$US9917 more than the non-MDD cohort; all $p<0.001$ ). Interestingly, the adjusted all-cause healthcare cost difference for TRD versus non-TRD MDD patients in this study were higher than the cost difference reported by Amos et al. [8] (\$US9479 vs. 6709). Given our IDN definition, the difference in costs may result from linking outpatient and inpatient institutions, which can increase the costs of both outpatient and inpatient care, as suggested by previous literature on IDNs [33]. Another potential explanation to account for this difference could be the continuity of care provided 


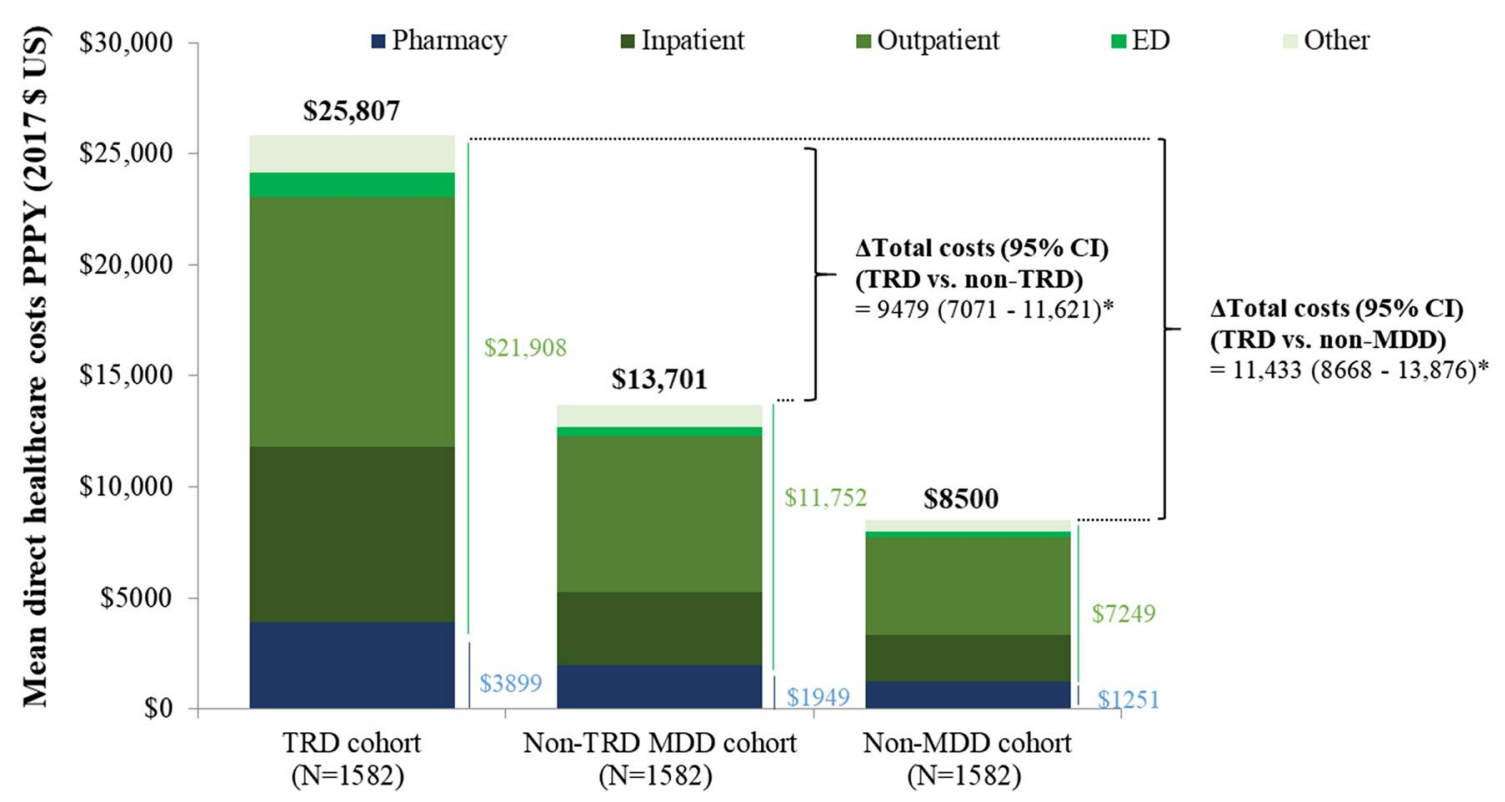

Fig. 3 All-cause healthcare costs per patient per year measured from the index date up to 2 years post-index date ${ }^{\mathrm{a}, \mathrm{b}}$. ${ }^{\mathrm{a}}$ Multivariable ordinary least squares regression included baseline all-cause healthcare costs and Quan-CCI; ${ }^{\mathrm{b}}$ Total costs are presented in the black text. Medical and pharmacy costs are presented in the green and blue texts,

by healthcare providers in IDNs resulting in an increased awareness of patients' worsening symptoms, which would require additional care and ultimately contribute to greater HRU and costs.

To the best of our knowledge, this is the first study to highlight treatment patterns, HRU, and costs associated with TRD, non-TRD MDD, and non-MDD patients treated in US IDNs. As a result, direct comparisons with other studies are a challenge because of the differences in study parameters and patient populations evaluated. However, one study published in 2003, by Liu et al. [34], examined the cost effectiveness of collaborative care for veterans with MDD and reported an increase in costs associated with collaborative care. As expected, their explanation for the higher costs were the greater resources needed to adequately treat MDD patients. Despite methodological differences between that study and the present work, it is reasonable to conclude that the high HRU and costs pertain to the greater needs of the TRD patient population. The HRU and costs among the cohorts in this study provide a useful benchmark to help direct efforts in reducing the health and economic burdens associated with MDD, particularly TRD. Consequently, this study fills an important knowledge gap for IDN decision makers regarding real-world treatment patterns and cost differences for TRD patients, which significantly contributes to the overall burden of MDD.

Although the results of this study do not support lower costs among TRD or non-TRD MDD patients treated in respectively. *Significant at the $5 \%$ level. $C I$ confidence interval, $E D$ emergency department, $M D D$ major depressive disorder, $P P P Y$ per patient per year, Quan-CCI Quan-Charlson comorbidity index, TRD treatment-resistant depression

IDNs, it is possible that the true benefit of IDNs among these patient populations is in the quality of care received. Given the care coordination among providers across a continuum of settings, IDNs are particularly relevant for patients with behavioral health disorders $[19,35]$. Several studies have documented improved patient outcomes within IDNs for patients with depressive disorders [36-39]. For example, one study based on longitudinal data found that patients treated in highly integrated programs had greater improvement in self-reported physical health and mental health recovery assessed by their clinician [40]. Another study that compared outcomes among patients with depression who received collaborative care and those who received usual care found that collaborative care was associated with significant improvement in adherence to therapy ( 75 vs. $50 \%$ ), patient perception of quality of care (93 vs. 75\%), and patient-reported improvement in efficacy of antidepressant therapy (88 vs. 63\%) [36]. The fact that several studies highlight improved quality of care among heterogeneous patient populations underscores the value of IDNs. It is possible that the higher quality of care may result in reduced costs long term; however, additional studies are warranted to explore this possibility among TRD, non-TRD MDD, and non-MDD patients treated across different types of IDNs versus non-IDN settings. 


\subsection{Limitations}

This study should be interpreted within the context of certain limitations. First, the treatment failure algorithm used to identify patients with TRD relied solely on pharmacy claims; clinical considerations to specifically assess treatment failure, response, and remission could not be incorporated. Furthermore, the algorithm used to identify the TRD study population may not be representative of all TRD patients as only patients with an adequate dose and duration of antidepressant were selected. Second, because patients with a single IDN visit were included in the analysis, it is possible that some of the patients evaluated did not experience the care associated with multiple visits to an IDN. Additionally, once patients are seen in an IDN, they remain flagged as IDN patients even if they later receive care in a non-IDN setting. Although this may prevent us from accurately identifying patients who are truly seen in an IDN setting, we hypothesized that patients seen in an IDN typically prefer to continue their care within the same IDN in which they were initially seen based on available literature on patient experience in IDNs [33, 41]. Third, PS matching based on demographic variables was conducted to minimize the confounding between the TRD and non-TRD MDD or non-MDD cohorts. However, since imbalances remained after matching, we further adjusted for baseline Quan-CCI and all-cause healthcare costs. Although PS matching and adjustment techniques were used to minimize the potential confounding, these comparisons may still be subject to residual confounding from unmeasured confounders. Fourth, to evaluate baseline characteristics and study outcomes, a minimum of 6 months of continuous enrollment in a health insurance plan before and after the index date was imposed on the study population. However, patients who do not have $\geq 6$ months of continuous enrollment before or after the index date may have differed in the continuity of care received. This may limit the generalizability of the HRU and cost burden reported in this study. Lastly, there is currently no industry standard to define an IDN [15]. In this study, an IDN was defined as a coordinated hospital inpatient and outpatient system offering healthcare services in a defined geographical region. Therefore, the results may not be generalizable to all US IDNs.

\section{Conclusions}

Patients with TRD who received care in IDNs had higher HRU and incremental costs than patients with MDD who were not resistant to treatment and those without MDD. The HRU and economic burden associated with TRD were substantial in the US IDN setting and was mainly driven by non-behavioral health-related HRU and costs.
Acknowledgements Medical writing assistance was provided by Gloria DeWalt, an employee of Analysis Group, Inc. Support for this assistance was provided by Janssen Scientific Affairs, LLC.

Author Contributions HS, KJ, DS, and JJS contributed to the conception and design of the study and data analysis and interpretation. DP, JWW, PL, and PG contributed to the conception and design of the study, acquisition of data, and data analysis and interpretation.

Data Availability The data supporting the findings of this study are available from Optum. Restrictions apply to the availability of these data. Data were used under license for the current study and are not publicly available.

\section{Compliance with Ethical Standards}

Funding This study was funded by Janssen Scientific Affairs, LLC.

Conflict of interest DP, JWW, PL, and PG are employees of Analysis Group, Inc, which received consultancy fees from Janssen Scientific Affairs, LLC for the conduct of this study. HS, KJ, and JJS are employees of Janssen Scientific Affairs, LLC. KJ is also a stockholder of Johnson \& Johnson. DS was a post-doctoral fellow at Thomas Jefferson University.

Open Access This article is distributed under the terms of the Creative Commons Attribution-NonCommercial 4.0 International License (http://creativecommons.org/licenses/by-nc/4.0/), which permits any noncommercial use, distribution, and reproduction in any medium, provided you give appropriate credit to the original author(s) and the source, provide a link to the Creative Commons license, and indicate if changes were made.

\section{References}

1. Mayo Clinic. Depression (major depressive disorder). 2018. https ://www.mayoclinic.org/diseases-conditions/depression/symptomscauses/syc-20356007. Accessed 06 Aug 2018.

2. Hasin DS, Sarvet AL, Meyers JL, Saha TD, Ruan WJ, Stohl M, et al. Epidemiology of adult DSM-5 major depressive disorder and its specifiers in the United States. JAMA Psychiatry. 2018;75(4):336-46. https://doi.org/10.1001/jamapsychi atry.2017.4602.

3. National Institute of Mental Health (NIMH). Major depression. 2017. https://www.nimh.nih.gov/health/statistics/major-depre ssion.shtml. Accessed 06 Jul 2018.

4. Greenberg PE, Fournier AA, Sisitsky T, Pike CT, Kessler RC. The economic burden of adults with major depressive disorder in the United States (2005 and 2010). J Clin Psychiatry. 2015;76(2):15562. https://doi.org/10.4088/JCP.14m09298.

5. Fava M, Davidson KG. Definition and epidemiology of treatmentresistant depression. Psychiatr Clin N Am. 1996;19(2):179-200.

6. Kubitz N, Mehra M, Potluri RC, Garg N, Cossrow N. Characterization of treatment resistant depression episodes in a cohort of patients from a US commercial claims database. PLoS One. 2013;8(10):e76882. https://doi.org/10.1371/journal.pone.00768 82.

7. American Psychiatric Association. Practice guideline for the treatment of patients with major depressive disorder third Edition. 2010. https://psychiatryonline.org/pb/assets/raw/sitewide/pract ice_guidelines/guidelines/mdd.pdf. Accessed 27 Jun 2018. 
8. Amos TB, Tandon N, Lefebvre P, Pilon D, Kamstra RL, Pivneva $\mathrm{I}$, et al. Direct and indirect cost burden and change of employment status in treatment-resistant depression: a matched-cohort study using a US commercial claims database. J Clin Psychiatry. 2018. https://doi.org/10.4088/jcp.17m11725.

9. Olfson M, Amos TB, Benson C, McRae J, Marcus SC. Prospective service use and health care costs of medicaid beneficiaries with treatment-resistant depression. J Manag Care Spec Pharm. 2018;24(3):226-36.

10. Ivanova JI, Birnbaum HG, Kidolezi Y, Subramanian G, Khan SA, Stensland MD. Direct and indirect costs of employees with treatment-resistant and non-treatment-resistant major depressive disorder. Curr Med Res Opin. 2010;26(10):2475-84. https://doi. org/10.1185/03007995.2010.517716.

11. Enthoven AC. Integrated delivery systems: the cure for fragmentation. Am J Manag Care. 2009;15(10 Suppl):S284-90.

12. Peek CJ, Cohen DJ, deGruy FV 3rd. Research and evaluation in the transformation of primary care. Am Psychol. 2014;69(4):430-42. https://doi.org/10.1037/a0036223.

13. Trivedi MH, Daly EJ. Measurement-based care for refractory depression: a clinical decision support model for clinical research and practice. Drug Alcohol Depend. 2007;88(Suppl 2):S61-71. https://doi.org/10.1016/j.drugalcdep.2007.01.007.

14. Lake J, Turner MS. Urgent need for improved mental health care and a more collaborative model of care. Perm J. 2017. https:// doi.org/10.7812/tpp/17-024.

15. Hwang W, Chang J, Laclair M, Paz H. Effects of integrated delivery system on cost and quality. Am J Manag Care. 2013;19(5):e175-84.

16. Bao Y, Casalino LP, Pincus HA. Behavioral health and health care reform models: patient-centered medical home, health home, and accountable care organization. J Behav Health Serv Res. 2013;40(1):121-32. https://doi.org/10.1007/s1141 4-012-9306-y.

17. Rittenhouse DR, Casalino LP, Gillies RR, Shortell SM, Lau B. Measuring the medical home infrastructure in large medical groups. Health Aff (Millwood). 2008;27(5):1246-58. https://doi. org/10.1377/hlthaff.27.5.1246.

18. Alakeson V, Frank RG, Katz RE. Specialty care medical homes for people with severe, persistent mental disorders. Health Aff (Millwood). 2010;29(5):867-73. https://doi.org/10.1377/hltha ff. 2010.0080 .

19. Jones AL, Mor MK, Haas GL, Gordon AJ, Cashy JP, Schaefer JH $\mathrm{Jr}$, et al. The role of primary care experiences in obtaining treatment for depression. J Gen Intern Med. 2018;33(8):1366-73. https ://doi.org/10.1007/s11606-018-4522-7.

20. LexisNexis Risk Solutions. The ABCs of IDNs: the new keys to commercial success for Life Sciences Organizations. 2016. https ://risk.lexisnexis.com/-/media/files/healthcare/white-paper/abcsidn-wp-pdf.pdf. Accessed 30 Aug 2018.

21. Datamonitor Healthcare. Integrated Delivery Networks in the US-Stakeholder interest in integrated delivery networks is growing. 2016. https://pharmaintelligence.informa.com/ /media /Informa-Shop-Window/Pharma/Files/Reports/Integrated-Deliv ery-Networks-in-the-US-TOC-June-2017-1.pdf. Accessed 30 Aug 2018.

22. Whitebird RR, Solberg LI, Jaeckels NA, Pietruszewski PB, Hadzic S, Unutzer J, et al. Effective implementation of collaborative care for depression: what is needed? Am J Manag Care. 2014;20(9):699-707.

23. Dickinson LM, Rost K, Nutting PA, Elliott CE, Keeley RD, Pincus $\mathrm{H}$. RCT of a care manager intervention for major depression in primary care: 2-year costs for patients with physical vs psychological complaints. Ann Fam Med. 2005;3(1):15-22. https://doi. org/10.1370/afm.216.
24. Grochtdreis T, Brettschneider C, Wegener A, Watzke B, RiedelHeller S, Harter M, et al. Cost-effectiveness of collaborative care for the treatment of depressive disorders in primary care: a systematic review. PLoS One. 2015;10(5):e0123078. https://doi. org/10.1371/journal.pone.0123078.

25. Gartlehner G, Gaynes BN, Amick HR, Asher G, Morgan LC, Coker-Schwimmer E et al. Nonpharmacological versus pharmacological treatments for adult patients with major depressive disorder. AHRQ Comp Effect Rev. 2015.

26. Austin PC. An introduction to propensity score methods for reducing the effects of confounding in observational studies. Multivar Behav Res. 2011;46(3):399-424. https://doi.org/10.1080/00273 171.2011.568786.

27. Pan W, Bai H. Propensity score analysis: concepts and issues. Propensity score analysis: fundamentals and developments. 2015;3-19.

28. Wijeysundera HC, Wang X, Tomlinson G, Ko DT, Krahn MD. Techniques for estimating health care costs with censored data: an overview for the health services researcher. Clinicoecon Outcomes Res. 2012;4:145-55. https://doi.org/10.2147/CEOR.S3155 2.

29. Quan H, Sundararajan V, Halfon P, Fong A, Burnand B, Luthi JC, et al. Coding algorithms for defining comorbidities in ICD-9-CM and ICD-10 administrative data. Med Care. 2005;43(11):1130-9.

30. Melek SP, Norris DT, Paulus J, Matthews K, Weaver A, Davenport S. Potential economic impact of integrated medical-behavioral healthcare: updated projections for 2017. Milliman, Inc. Prepared for American Psychiatric Association. 2017. http://www.milliman. com/insight/2018/Potential-economic-impact-of-integrated-medic al-behavioral-healthcare-Updated-projections-for-2017/. Accessed 08 Apr 2019.

31. Olchanski N, McInnis Myers M, Halseth M, Cyr PL, Bockstedt L, Goss TF, et al. The economic burden of treatment-resistant depression. Clin Ther. 2013;35(4):512-22. https://doi.org/10.1016/j.clint hera.2012.09.001.

32. Pilon D, Sheehan JJ, Szukis H, Singer D, Jacques P, Lejeune D, et al. Medicaid spending burden among beneficiaries with treatment-resistant depression. J Comp Eff Res. 2019;8(6):381-92. https://doi.org/10.2217/cer-2018-0140.

33. Burns LR, Pauly MV. Integrated delivery networks: a detour on the road to integrated health care? Health Aff (Millwood). 2002;21(4):128-43. https://doi.org/10.1377/hlthaff.21.4.128.

34. Liu CF, Hedrick SC, Chaney EF, Heagerty P, Felker B, Hasenberg $\mathrm{N}$, et al. Cost-effectiveness of collaborative care for depression in a primary care veteran population. Psychiatr Serv. 2003;54(5):698704. https://doi.org/10.1176/appi.ps.54.5.698.

35. Unutzer J, Park M. Strategies to improve the management of depression in primary care. Prim Care. 2012;39(2):415-31. https ://doi.org/10.1016/j.pop.2012.03.010.

36. Katon W, Von Korff M, Lin E, Walker E, Simon GE, Bush T, et al. Collaborative management to achieve treatment guidelines. Impact on depression in primary care. JAMA. 1995;273(13):1026-31.

37. Katon W, Von Korff M, Lin E, Simon G, Walker E, Unutzer J, et al. Stepped collaborative care for primary care patients with persistent symptoms of depression: a randomized trial. Arch Gen Psychiatry. 1999;56(12):1109-15.

38. Hunkeler EM, Katon W, Tang L, Williams JW Jr, Kroenke $\mathrm{K}$, Lin $\mathrm{EH}$, et al. Long term outcomes from the IMPACT randomised trial for depressed elderly patients in primary care. BMJ. 2006;332(7536):259-63. https://doi.org/10.1136/bmj.38683 .710255.BE.

39. Butler M, Kane RL, McAlpine D, Kathol RG, Fu SS, Hagedorn $\mathrm{H}$, et al. Integration of mental health/substance abuse and primary care. Evid Rep Technol Assess (Full Rep). 2008;173:1-362.

40. Gilmer TP, Henwood BF, Goode M, Sarkin AJ, Innes-Gomberg D. Implementation of integrated health homes and health outcomes 
for persons with serious mental illness in Los Angeles County. Psychiatr Serv. 2016;67(10):1062-7. https://doi.org/10.1176/appi. ps.201500092.

41. O'Malley AS, Cunningham PJ. Patient experiences with coordination of care: the benefit of continuity and primary care physician as referral source. J Gen Intern Med. 2009;24(2):170-7. https:// doi.org/10.1007/s11606-008-0885-5.

42. Elixhauser A, Steiner C, Kruzikas. D. HCUP Methods Series Report \# 2004-1. 06 February 2004.
43. US Agency for Healthcare Research and Quality. Comorbidity Software Documentation. Rockville, MD, USA; 2004. p. 12-5. http://www.hcup-us.ahrq.gov/reports/ComorbiditySoftwareD ocumentationFinal.pdf. Accessed 2013.

44. American Psychiatric Association. Diagnostic and statistical manual of mental disorders: DSM-V. Amer Psychiatric Pub Inc; 2013.

\section{Affiliations}

\section{Dominic Pilon ${ }^{1}$ (I) Holly Szukis ${ }^{2} \cdot$ Kruti Joshi $^{2} \cdot$ David Singer $^{3} \cdot$ John J. Sheehan ${ }^{2} \cdot$ Jennifer W. Wu $^{1} \cdot$ Patrick Lefebvre $^{1}$. Paul Greenberg ${ }^{4}$}

Holly Szukis

HSzukis1@its.jnj.com

Kruti Joshi

KJoshi33@its.jnj.com

David Singer

dasinger137@gmail.com

John J. Sheehan

Jsheeha2@its.jnj.com

Jennifer W. Wu

Jennifer.Wu@analysisgroup.com

Patrick Lefebvre

Patrick.Lefebvre@analysisgroup.com
Paul Greenberg

Paul.Greenberg@analysisgroup.com

1 Analysis Group, Inc., 1190 avenue des

Canadiens-de-Montreal, Tour Deloitte Suite 1500, Montréal, QC H3B 0G7, Canada

2 Janssen Scientific Affairs, LLC, Titusville, NJ, USA

3 Thomas Jefferson University, Philadelphia, PA, USA

4 Analysis Group, Inc., Boston, MA, USA 